\title{
Wear Resistance of Steels under Conditions of Dry Sliding Friction and Electric Current of High Density
}

\author{
Viktor V. Fadin ${ }^{1, \text { a) }}$, Marina I. Aleutdinova ${ }^{1, \text { b) }}$, Aleksandr V. Kolubaev ${ }^{1,2, c)}$, \\ Valeriya A. Aleutdinova, d) \\ ${ }^{1}$ Institute of Strength Physics and Materials Science SB RAS, Tomsk, 634055, Russia \\ ${ }^{2}$ National Research Tomsk Polytechnic University, Tomsk, 634050, Russia \\ ${ }^{3}$ National Research St. Petersburg State Polytechnical University, St. Petersburg, 195251, Russia \\ a) Corresponding author: fvv@ispms.ru \\ b) aleut@ispms.ru \\ c) kav@ispms.ru \\ d) valery-aleut@yandex.ru
}

\begin{abstract}
The dependence of current density on the conductivity and the wear rate for the electric contact steel/steel 45 under conditions of dry sliding friction at current density higher $100 \mathrm{~A} / \mathrm{cm}^{2}$ has been presented. It has been determined that the increase in the content of alloying elements or reinforcing phase in the initial structure of the steel leads to lower strength of the surface layer, resulting in a high wear rate of contact and catastrophic wear at a low current density. It has been noted that the rapid destruction of the surface layer of alloy steels is caused by their low plasticity limits. The high values of wear resistance of non-alloyed steel at friction with current collection are due to the instability to the rotary and shear deformation, as well as to the formation of a viscous fluid on the sliding surface.
\end{abstract}

Keywords: fragmentation of surface layer, rotational plastic deformation, shear instability of material, wear resistance, structure of surface layer, phase composition.

\section{INTRODUCTION}

It is known that plastic deformation is caused by the local loss of solid shear stability and starts in the tension concentration zones of respective scale. As a result, coupled tensions appear, its relaxation causes rotational deformation modes emergence that are responsible for the formation of mesostructure of lower level—fragmented substructure. It leads to vortex motion pattern of mesovolumes and the appearance of local discontinuity flaws and microcracks, which is completed by the main crack propagation and the emergence of wear particle in case of surface layer deformation under sliding friction. In doing so, wear particles are mesovortexes of deformation and deterioration in near-surface friction layers. It is necessary to block mesovortexes initiation for wear rate reduction [1]. As a result, the surface layer strength can be increased by the deceleration of wear particle formation process, i.e. by surface layer fragmentation suppression or by fragmentation process compensation. One can suppress fragmentation by strengthening surface layer, for example by implantation, deposition of coating etc. It is possible to compensate fragmentation due to using materials with low shearing resistance; there is a possibility of phasestructural transformation under load and quasiviscous flowing of material between misoriented fragments, when well coordinated hierarchy of gradient rotational deformation modes propagates. Then high fracture toughness of surface layer and possibility of its nanostructuring are implemented that leads to high wear resistance [2]. These two methods of effect on fragmentation generally are applied to produce an efficient triboengineering material. It is of a special interest to generate a material intended to work under extreme external loading. The main stress incipient in tribocontact of materials is in the spots of real contact. Deformation of microvolumes adjacent to contact spots leads to thermal flare, sharp enhancement of diffusion coefficient, high rate of heat dissipation. In this case one should 
choose a method of influence on surface layer fragmentation. Extreme external coercion on contact spots could be generated by friction and current flow where current density is the main damaging factor. Wear rate can be the indicator of surface layer strength at the macrolevel. Steels with diverse compositions can be model materials for such a type of loading. Steel composition is a parameter that controls surface layer plasticity and wear rate.

The aim of the present work is to get the initial idea of relationship between the element and phase composition of the initial structure and the intensity of steels wear under friction tests at current streaming trough contact surface and at contact current density higher $100 \mathrm{~A} / \mathrm{cm}^{2}$.

\section{EXPERIMENTAL DETAILS}

Steels that served as model materials were obtained using standard manufacturing technologies. The samples of high-speed steel (HSS), ball bearing steel, Hadfield steel (13\% Mn) were in quenched state. Phase composition of the surface layer was determined using a DRON-3 X-ray diffractometer. Metallographic examination was carried out on Neophot-21 optical microscope. Steels were tested by dry sliding contact under alternating current $(50 \mathrm{~Hz})$, pressure $0.13 \mathrm{MPa}$, sliding velocity $5 \mathrm{~m} / \mathrm{s}$ on tribometer. Tests were conducted using "blockon-shaft" scheme (Fig. 1(a)). Steel $45(0.45 \%$ C, 50 HRC) was used as a counterbody. Sliding distance was $9 \mathrm{~km}$. Linear wear intensity was determined $I_{h}=h / L$, where $h$ is alteration of height of sample on sliding distance $L$.

\section{RESULTS AND DISCUSSION}

Contact current density $j=i / A_{\mathrm{a}}$ depends on electrical contact resistance $\mathrm{r}$ and is defined by structural state of surface layer $\left(i\right.$ - current carried through nominal contact area $\left.A_{\mathrm{a}}\right)$. Electric conductivity $r_{\mathrm{s}}^{-1}=j / U(U-$ contact potential drop) of the steels contact increases in a certain limit of contact current density $j$ values (Fig. 1(b)). Maximum of electric contact conductivity corresponds to point of inflection in a current-voltage diagram and sharp increase in wear intensity $I_{h}$ (Fig. 1(c)) that indicates the catastrophic wear onset under a certain value $j=j_{\mathrm{c}}$. One can see that high speed steel containing several phases can not correspond to this regularity because of high wear under low current density. Contact characteristics of steel 3 are higher than those of other steels (Fig. 1(b, c)). Moreover, contact current density $j_{\mathrm{c}}$ is higher in friction of steel 3 than $j_{\mathrm{c}}$ of other steels. In addition, one can see that when concentration of alloying elements or phases in the initial structure increases, wear resistance of contact decreases, which is conditioned by plasticity and structure of surface layer.

Plastic deformation of surface layer $L$ causes its structural changes that can be observed as the formation of friction induced structures (FIS) layer. One can see that FIS-layer of steel 3 is rather thin (white color layer) and has small amount of discontinuity flaws (Fig. 2(a)). FIS layer of ball bearing steel has the image close to FIS layer of steel 3 (Fig. 2(c)). Increase in the number of the alloying elements or phases in the initial structure of HSS and Hadfield steels leads to the formation of thick layer of FIS with considerable number of discontinuity flaws (Fig. 2(e, g). It should be noted that sliding surface of steel 3 (рис. 2(b)) has explicit indications of liquid phase formation, while the same indications are not so clearly defined on sliding surface of other steels. Contact characteristics and plasticity of surface layer substantially depend on the phase structure of FIS. It has been shown in the present paper that the FIS layers contain BCC-iron and FCC-iron as well as FeO oxide (Table 1).
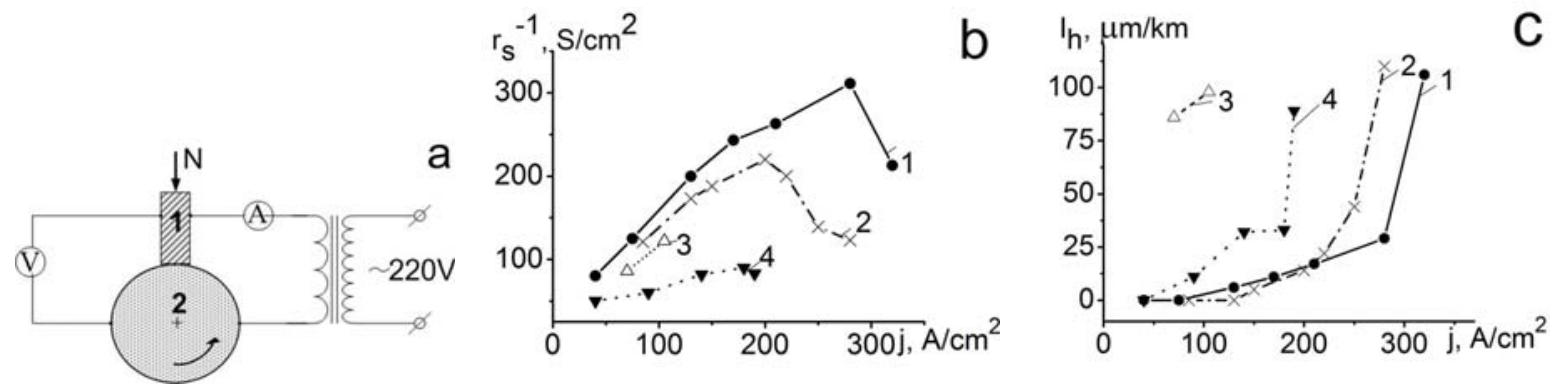

FIGURE 1. Basic scheme "block-on-shaft" of tribotests with sliding current collection: 1 - specimen, 2 -conterbody (steel 45) (a); current dependence of specific surface electric conductance (b) and wear intensity (c) of contact of steel $3(\bullet)$, ball bearing steel $(\times)$, HSS $(\Delta)$, Hadfield steel $(\boldsymbol{\nabla})$ 

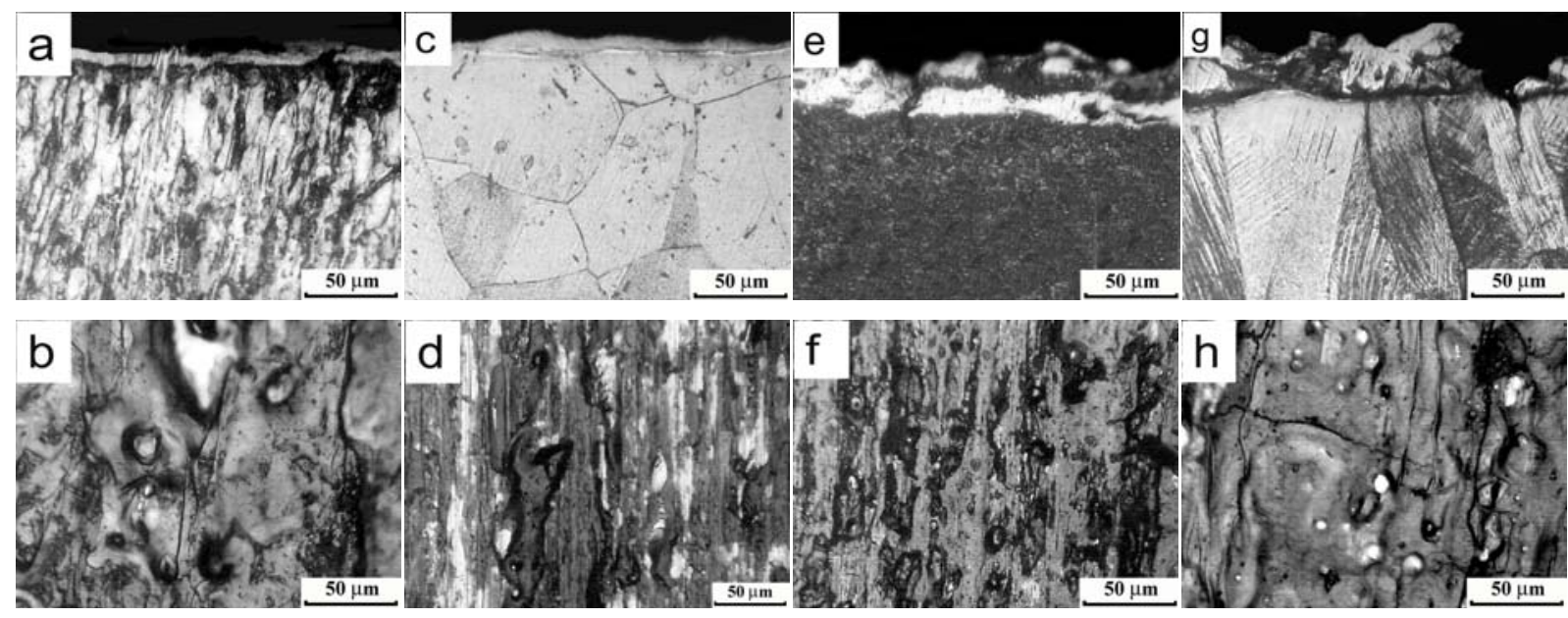

FIGURE 2. Initial structure and sliding surface profile of steel 3(a), ball bearing steel (c), HSS (e), Hadfield steel (g) and worn surface of steel 3 (b), ball bearing steel (d), HSS (f), Hadfield steel (h)

One can assume that FIS contains quasiamorphous phase as well.

Implementation of $I_{\mathrm{h}}>0$ indicates that contact spots deform plastically under the above stated loading condition. It is known that addition of alloying atoms or additional crystal phases serves to decrease the plasticity limit [3]. This initial structure has to reduce plasticity of FIS, i.e. decrease the ability to stress relaxation in surface layer. Steel $3(0.2 \% \mathrm{C},>98 \% \mathrm{Fe})$ does not have alloying atoms. It allows to have a satisfactory plastic limit and to form FIS being unstable to rotation and shear. Rather high plasticity of these FIS and its ability to be deformed similar to viscous fluid pattern lead to lower scale of stress localization that causes the development of mesobands of plastic deformation and generation of structural defects expanding inwards the sample. This promotes precipitation of small scale defects and formation of thin FIS layer with minor amount of defects. Furthermore, the absence of alloying leads to high thermal conductivity $\lambda$ and low specific resistance $\rho$ (Table 1). These factors assist to the increase in wear resistance and $j_{\mathrm{c}}$ that corresponds to the beginning of catastrophic wear. High concentration of doping elements or phases in Hadfield steel $(\mathrm{Fe}+13 \% \mathrm{Mn})$ and $\mathrm{HSS}(\mathrm{Fe}+6 \% \mathrm{~W}+5 \% \mathrm{Mo}+$ carbides) causes growth of specific resistance $\rho$ and decrease in thermal conductance $\lambda$ (Table 1) that leads to high local temperature gradients. Besides, alloying elements reduce plasticity of original structure and cause the increase in local shear stability that opposes to compensation of rotational modes under conditions of inescapable plastic deformation of surface layer. As a result, plastic shears occur at larger depth, FIS layer is thick and power mesoconcentrators of stresses appear. Weak stress relaxation arising as a result of rotational deformation leads to crack formation in FIS layer (Fig. 2(e, g)) and to high wear under low contact current density.

Table 1. Main parameters of zone of steel sliding electric contact at onset of catastrophic wear

\begin{tabular}{lcccc}
\hline \multicolumn{1}{c}{ PropertylSteel } & Steel 3 & Ball Bearing Steel & HSS & Hadfield Steel \\
\hline $\mathrm{HB}, \mathrm{MPa}$ & 1360 & $63 \mathrm{HRC}$ & $64 \mathrm{HRC}$ & 2430 \\
\hline$P, 10^{-6} \mathrm{OHM} \mathrm{M}$ & 0.2 & 0.29 & 0.46 & 0.67 \\
\hline$\Lambda, \mathrm{W} / \mathrm{M} \mathrm{K}$ & 55 & 43 & 28 & 11 \\
\hline$A_{\mathrm{FCC}-\mathrm{FE}}, \mathrm{NM}$ & 0.3595 & 0.3604 & 0.3609 & 0.3601 \\
\hline$A_{\mathrm{FEO}}, \mathrm{NM}$ & 0.4307 & 0.4303 & 0.4301 & 0.4330 \\
\hline$A_{\mathrm{BCC}-\mathrm{FE}}, \mathrm{NM}$ & 0.2867 & 0.2867 & 0.2867 & 0.2875 \\
\hline$R_{\mathrm{SC}}{ }^{-1}, \mathrm{~S} / \mathrm{CM}^{2}$ & 320 & 220 & $<50$ & 75 \\
\hline$J_{\mathrm{C}}, \mathrm{A} / \mathrm{CM}^{2}$ & 275 & 200 & $<50$ & 0.62 \\
\hline$A_{\mathrm{FCC}}$ & 0.251 & 0.084 & - & 577 \\
\hline$T,{ }^{\circ} \mathrm{C}$ & 326 & 306 & & 0.871 \\
\hline
\end{tabular}


This information indicates that complex initial structure, i.e. presence of solid solutions or high number of phases causes low thermal conductivity and low plasticity of surface layer. Sliding contact of such material can realize minor wear only at low temperature of the friction zone and under low magnitude of basic parameter (pressure, sliding velocity etc.) of external action which is responsible for growth of surface layer temperature and its destruction.

High magnitude of mass ratio of FCC-iron to amount FeO oxide (parameter $A_{\mathrm{FCC}}=I_{1} /\left(I_{2}+I_{3}\right)$ ) indicates high relative amount of FCC-iron in FIS of HSS and Hadfield steel (Table 1) that corresponds to low wear resistance (here $I_{1}$ - integral intensity of the most intense reflection of (111) FCC-iron, $\left(I_{2}+I_{3}\right)$-integral intensity of the most intense reflection of (111) and (200) $\mathrm{FeO}$ oxide). Evidently, wear resistance increases in the absence of solid solutions and large number of phases in the initial structure and in FIS, and also at low content of FCC-iron in FIS and ability to form liquid phase at sliding surface.

One can assume that the formation of viscous fluid on frictional surface occurs as a result of local melting of the surface layer. It is of an interest to estimate the average temperature of surface layer at the stage of transition to catastrophic wear. The calculation was based on Fourier's law. Coefficient of thermal currents distribution was obtained using Block formula. It has been found out that temperature $T$ of sliding surface (table) is noticeably lower than the melting temperature of steel. Electric discharge is able to melt surface layer only locally. Therefore, there should be another reason for the appearance of liquid phase in the contact layer. Common observations show that the appearance of viscous fluid is connected with increase in mobility and velocity of atoms in surface layer. It is known that mesovortex arise in plastic deformation where the rate of mass transfer could increase up to 15 orders of magnitude as compared with regular diffusion [4]. One can assume that noticeable growth of atoms mobility is caused by appearing mesovortices in surface layer. Very fast diffusion could occur as well when considerable concentration of vacancies is generated under electric current. Such rotational dynamic deformation occurs easily in materials with original structure without signs of strengthening, i.e. without alloying, strengthening phases etc. Then FIS of such materials must be unstable at rotation and shear plastic deformation that can be seen by the example of plastic steel 3. Thus, wear-resistant sliding contacts under contact current density higher $100 \mathrm{~A} / \mathrm{cm}^{2}$ have to be composed of materials able to form shear changeable surface layer and liquid phase on sliding surface. Purposive introduction of liquid metals into the contact space also leads to some increase in wear resistance. However, this method of obtaining liquid phase on frictional surface is not effective or perspective [5]. It should also be mentioned (Table 1 and Fig. 1) that hardness of steels does not affect on contact characteristics.

\section{CONCLUSION}

Sliding with current collection at contact current density higher $100 \mathrm{~A} / \mathrm{cm}^{2}$ causes inevitable plastic deformation of surface layer that leads to the formation of a structurally modified layer. Wear resistance under such friction conditions would be high if this layer had low resistance to shear and rotational plastic deformation. The surface layer of steel under friction with current transfer fails fast if the initial structure is not complex, i.e. does not contain any signs of strengthening (solid solutions, high content of solid phases etc.).

\section{ACKNOWLEDGEMENT}

The work was carried out according to Program for Basic Scientific Research of the State Academy of Science for 2013-2020 and was supported by Russian Foundation for Basic Researches project No. 13-08-00076.

\section{REFERENCES}

1. V. E. Panin and P. A. Vityaz, Phys. Mesomech. 5(1), 5 (2002).

2. V. E. Panin, Yu. I. Pochivalov et al. Phys. Mesomech. 13(6), 27 (2010).

3. F. B. Pickering, Physical Metallurgy and the Design of Steels: Material Science Series (Applied Science Ppublishers LTD, 1978).

4. V. E. Panin and V. E. Egorushkin, Phys. Mesomech. 16(4), 267 (2013).

5. V. V. Fadin and M. I. Aleutdinova, J. Appl. Mech. Tech. Phys. 53(3), 432 (2012). 